\title{
Surgical treatment of GERD. Comperative study of WTP vs. Toupet fundoplication - results of 151 consecutive cases
}

\author{
Tadeusz Wróblewski, Konrad Kobryn, Małgorzata Nowosad, Marek Krawczyk \\ Department of General, Transplant and Liver Surgery, Medical University of Warsaw, Warsaw, Poland
}

Videosurgery Miniinv 2016; 11 (2): 60-66 DOI: $10.5114 /$ wiitm.2016.58947

\begin{abstract}
Introduction: Gastroesophageal reflux disease (GERD) is recognized as one of the most common disorders of the upper gastrointestinal tract (GIT). The best choice of management for advanced GERD is laparoscopic surgery.

Aim: To compare and evaluate the results of surgical treatment of GERD patients operated on using two different techniques.

Material and methods: Between 2001 and 2012, 353 patients (211 female and 142 male), aged 17-76 years (mean 44), underwent laparoscopic antireflux surgery. The study included patients who underwent a Toupet fundoplication or Wroblewski Tadeusz procedure (WTP).

Results: The mean age of the group was 47.77 years (17-80 years). Forty-nine (32.45\%) patients had severe symptoms, 93 (61.58\%) had mild symptoms and 9 (5.96\%) had a single mild but intolerable sign of GERD. Eighty-six (56.95\%) patients had a Toupet fundoplication and 65 (43.04\%) had a WTP. The follow-up period was 18-144 months. The average operating time for Toupet fundoplication and the WTP procedure was $164 \mathrm{~min}$ (90-300 min) and $147 \mathrm{~min}$ (90-210 min), respectively. The perioperative mortality rate was $0.66 \%$. The average post-operative hospitalization period was 5.4 days (2-16 post-operative days $(P O D)=$ Toupet) vs. 4.7 days $(2-9$ POD = WTP). No reoperations were performed. No major surgical complications were identified.

Conclusions: Wroblewski Tadeusz procedure due to a low percentage of post-operative complications, good quality of life of patients and a zero recurrence rate of hiatal hernia should be a method of choice.
\end{abstract}

Key words: gastroesophageal reflux disease, fundoplication, hiatal hernia.

\section{Introduction}

Gastroesophageal reflux disease (GERD) is a disorder comprising many factors. We cannot say about the epidemiology since the data found in the literature report from $1 \%$ to $39 \%$ of the population in well-developed countries [1-3]. Hiatal hernias which are symptomatic due to altered anatomy are the main and typical cause of GERD. The other factors, sometimes not well defined in individual patients, are: lower esophageal sphincter (LES) disorders, insufficient esophageal peristalsis, al- tered mucosal resistance for acid or rare alkaline contents, delayed emptying of the stomach, and antroduodenal motility disorders [3-6]. In addition to the above factors, altered anatomy involving changed and/or deteriorated hiatal crura, phrenoesophageal ligament and shortened esophagus could also play an important role in occurrence of GERD symptoms [6-8].

A hiatal hernia is described as the migration of the intra-abdominal contents (stomach, but also mesentery) into a widened diaphragmatic hiatus $[1,6,9,10]$, creating a hernia sac and causing the 
hiatal phrenoesophageal membrane together with other abdominal connective tissue to deteriorate the function of LES. In GERD patients hiatal hernias are found in up to $90 \%$ of surgically treated patients [9-12]. A number of classifications of hiatal hernias are used today, but the most common is the topographic description [2, 8, 9, 12]. However, having the patient diagnosed and examined in endoscopy, a very functional pre-operative endoscopic classification is used.

The accompanying gastrointestinal symptoms which impair the quality of life of GERD patients include epigastric pain, retrosternal burning, gas belching, dyspepsia, somatoform disorders and dysphagia. Other symptoms of lesser intolerance are coughing, hoarseness, shortness of breath, sore throat and burning feeling of the mouth and tongue. In most cases, heartburn can be relieved through diet and lifestyle changes; however, some people may require proton pump inhibitor treatment or eventually surgery. Recurrence and the severity of these symptoms cause patients to abandon pharmacological therapy and see a surgeon $[6,7,11]$. Currently the best choice of surgical management concerning GERD is considered to be laparoscopic surgery. An accepted and recommended treatment, advocated by the European Association of Endoscopic Surgery (EAES) at a consensus meeting in Vienna in 2013, of esophageal hiatal hernias in properly selected patients is laparoscopic fundoplication using routine and acknowledged methods (i.e. Nissen-Rossetti, Toupet, Dor). To date there are several methods used in a number of experienced centers, many of which have developed their own modifications.

The key role of every method is that it should not be worse, if not better than the formerly known methods, it should be safe for the patient, cost-effective and complication free. With these points taken into consideration we would have the ideal way to perform surgery. But as we all know, this is not always the case, and there is no "superb" method in any kind of surgery. In 2005 a study group in our department evaluated means of treatment and post-operative signs and symptoms in patients who underwent surgery due to GERD. The results then were acceptable, but did not satisfy the team just yet. This called for a "closer look" at diagnosis, preparation and surgery, as it was a time of search for improvement. In 2007 a new laparoscopic antireflux technique was performed, named after the sur- geon who introduced the method - the Wroblewski Tadeusz procedure (WTP) [9].

The positioning of the patient and insertion of trocars did not differ from the typical Toupet procedure, performed previously in the department $[8,9]$. The first two stages of the modification described herein are the same for all types of operations, such as Toupet or Nissen. The first stage comprises the dissection of the lower esophagus and the crura of the diaphragm in order to acquire a 5-6 cm in length ventral part of the esophagus and dissect "the window" under the esophagus (Photo 1) [8, 12]. The second stage is the suturing of the crura of the diaphragm - this is carried out routinely, and one or a maximum of two stitches are applied approximately $2 \mathrm{~cm}$ below the esophagus (Photo 2). The $3^{\text {rd }}$ stage relates to the modification of the operation and is based on applying to both sides of and under the esophagus a typical polypropylene mesh (SurgiPro - Covidien) on the sutured together crura of the diaphragm. This mesh is commonly used for inguinal hernia repairs and giant hiatal hernia. This typical for Lichtenstein technique mesh $(11 \times 6 \mathrm{~cm})$ is cut into two portions and next in one part of it an asymmetrical "u-shape" prosthesis is cut out with scissors starting from the upper edge in order to fit the esophagus [9] (Photo 3).

The mesh is then placed on the sutured crura with the wider arm on the right hand side. It is then attached to both the crura of the diaphragm using an Endohernia laparoscopic stapler (Covidien) or Se-

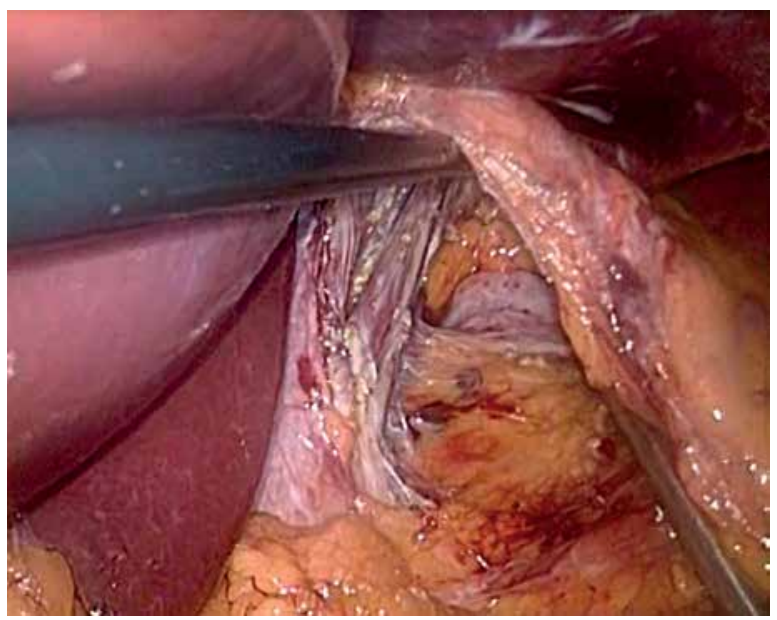

Photo 1. Dessected both cruras, ventrtal part of the oesophagus and "the window" under oesophagus 


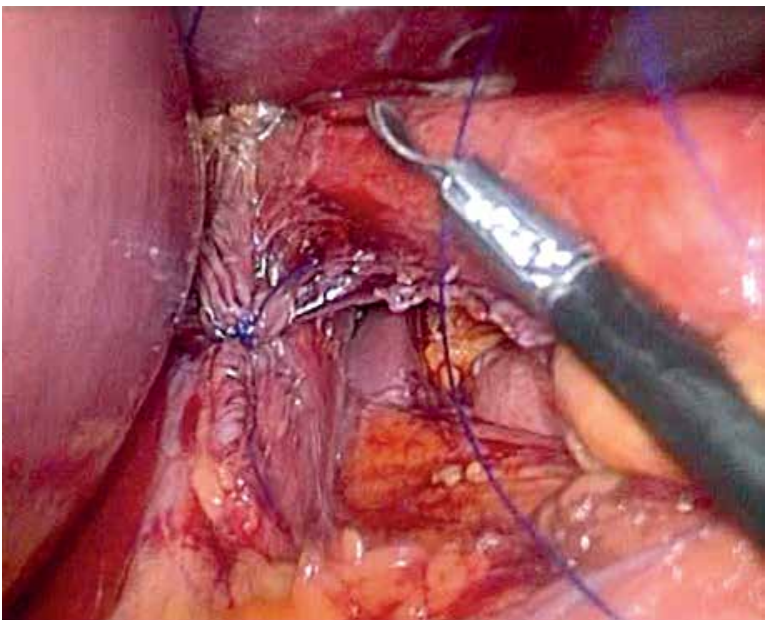

Photo 2. Stiched cruras of the hernia

curestrap (J\&J, USA) (Photo 4). The fourth and final stage of the modification is the restoration of a $90^{\circ}$ angle of His. In order to achieve this, the fundus of the stomach is sutured using three interrupted sutures to the left edge of the ventral part of the esophagus; a superior suture fixates the bottom of the stomach and esophagus with the diaphragm, protecting the whole plication from migrating to the mediastinum (Photo 5).

\section{Aim}

To compare and evaluate the results of treatment of 151 GERD patients after Toupet and WTP performed in the department who responded to Health-Related Quality of Life (HRQoL) questionnaires.

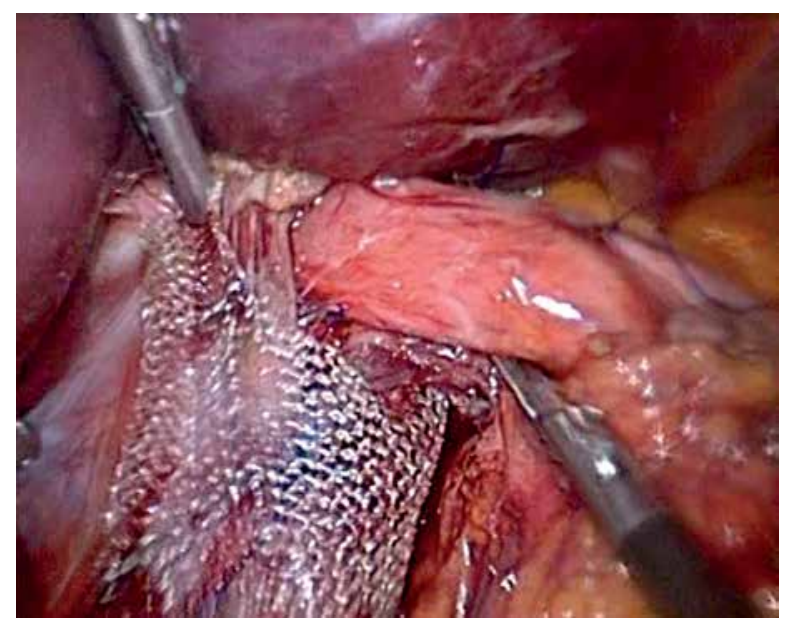

Photo 4. Stapled the U-mesh to the cruras

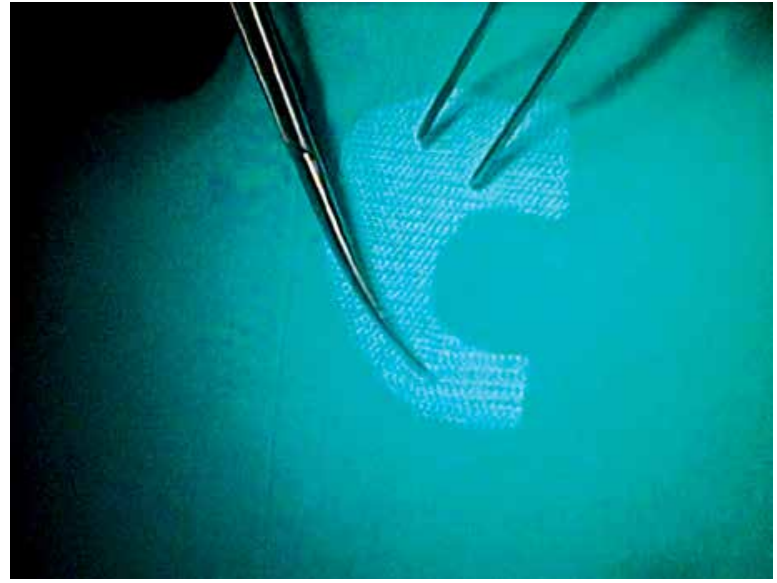

Photo 3. U-shaped polypropylen prosthesis for reinforced suturated cruras

\section{Material and methods}

We retrospectively analyzed medical data of 151 patients who underwent laparoscopic surgery during 2001-2012 in the Department of General, Liver and Transplant Surgery, Medical University of Warsaw, Poland. In the study we included patients who: previously had endoscopy and X-ray examination of the gastrointestinal tract with contrast media defining a preoperative diagnosis of gastroesophageal reflux disease, underwent a Toupet or WTP fundoplication, and prospectively answered HRQoL questionnaires.

We excluded from the study patients without complete pre-operative diagnosis of GERD, patients operated on by means of another technique (Nissen, Dor), and patients who failed to complete the HRQoL questionnaire.

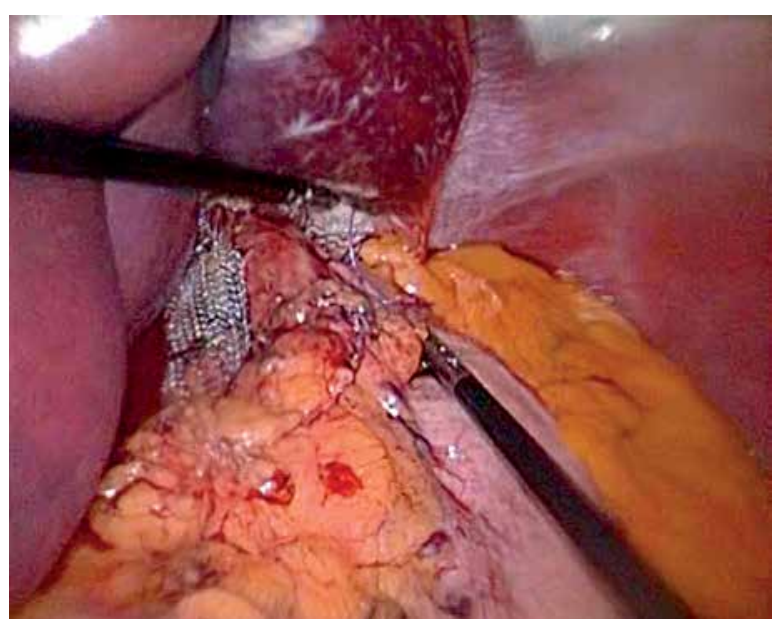

Photo 5. Complited WTP fundoplication 
Out of 151 GERD post-operative patients (POPs) there were 60 males and 91 females. The mean age of the group was 47.77 years (17-80 years). Forty-nine (32.45\%) POPs had severe symptoms of GERD such as acid reflux, dyspepsia, abdominal and epigastric pain and dysphagia. There were 93 (61.58\%) POPs with mild symptoms (coughing, mouth burning, shortness of breath) and 9 (5.96\%) POPs with a single mild but intolerable sign of GERD. In the group of operated patients 86 (56.95\%) had a Toupet fundoplication performed and 65 (43.04\%) patients underwent laparoscopic surgery using the WTP technique. The follow-up period ranged from 18 to 144 months. Full patient characteristics are found in Table I. All patients were operated on in the same conditions by the same surgical team, in one laparoscopic operating room, using the same brand laparoscopic instruments, with abdominal $\mathrm{CO}_{2}$ insufflation pressure of $15 \mathrm{~mm} \mathrm{Hg}$.

HRQoL questionnaires and a satisfaction survey were completed by patients, coded, ciphered and collected. The results were then implemented into an Excel database and analyzed statistically.

\section{Results}

During surgery, which initially always was to be laparoscopic, patients had a fundoplication performed. The hiatal hernia was identified each time in every procedure. The content of the hernia sac was most commonly dissected from the lower mediastinum and placed in the abdomen. The hiatal crura were sutured together in all patients sufficiently to close the hiatal openings but to allow free mobility of the esophagus and avoid post-operative complications such as dysphagia or return migration of fundoplication.

Table I. Group characteristics (cohort group: $n=151$ patients, male: $n=60$ vs. female: $n=91$ )

\begin{tabular}{|lc|}
\hline Parameter & Result \\
\hline Mean age [years] & $47.77(17-80)$ \\
\hline Severe preoperative symptoms, $n(\%)$ & $49(32.45)$ \\
\hline Mild preoperative symptoms, $n(\%)$ & $93(61.58)$ \\
\hline $\begin{array}{l}\text { Single but intolerable preoperative } \\
\text { symptom, } n \text { (\%) }\end{array}$ & $9(5.96)$ \\
\hline No. of Toupet fundoplications, $n(\%)$ & $86(56.95)$ \\
\hline No. of WTP, $n$ (\%) & $65(43.03)$ \\
\hline
\end{tabular}

There were two conversions to open laparotomy due to lack of possibilities of controlling arterial bleeding. Both conversions took place while performing the Toupet fundoplication. In 14 patients simultaneously a cholecystectomy was performed due to symptomatic gallbladder stones identified in abdominal ultrasound examination during the qualification for surgery. An extended range of surgery was conducted in 12 other patients simultaneously. All were performed laparoscopically along with the fundoplication: 1 resection of a small leiomyoma from the esophageal wall, 5 intraperitoneal onlay mesh (IPOM) hernioplasties of the linea alba in the epigastrium, 1 unroofing of a simple hepatic cyst, 1 wedge resection of a focal nodular hyperplasia (FNH) tumor in segment $V$ of the liver, 1 hernioplasty of the abdominal wall due to incisional hernia, 1 specimen collection of a tumor of the liver, 1 inguinal transabdominal preperitoneal (TAPP) hernioplasty with polypropylene mesh, 1 stapler resection of diverticula of the lower esophagus.

The average operating time by means of the Toupet fundoplication was $164 \mathrm{~min}$ (90-300 min), whereas that of WTP was $147 \mathrm{~min}$ (90-210 min). The perioperative mortality rate was $0.66 \%$, and concerned one 64-year-old female (Toupet procedure), where after one of the two conversions we made in the group in total, the patient died during surgery due to unexpected injury of an artery directly connecting to the aorta in the hiatus region, which caused uncontrolled hemorrhage and extensive blood loss. A comparison between the two methods during the follow-up period is presented in Table II.

The average post-operative hospitalization period for patients who underwent the Toupet operation was

Table II. Follow-up period (18-144 months)

\begin{tabular}{|c|c|c|}
\hline Parameter & $\begin{array}{c}\text { Toupet } \\
\text { fundoplication }\end{array}$ & WTP \\
\hline $\begin{array}{l}\text { Average operating time } \\
\text { [min] }\end{array}$ & $164(90-300)$ & $147(90-210)$ \\
\hline $\begin{array}{l}\text { Technical surgical } \\
\text { complications }\end{array}$ & No & No \\
\hline Early reoperation & No & No \\
\hline $\begin{array}{l}\text { Perioperative mortality } \\
\text { rate }\end{array}$ & $\begin{array}{l}1 \text { patients } \\
(0.66 \%)\end{array}$ & $\begin{array}{l}0 \text { patients } \\
(0 \%)\end{array}$ \\
\hline $\begin{array}{l}\text { Mean postoperative } \\
\text { hospitalization [days] }\end{array}$ & $5.4(2-16)$ & $4.7(2-9)$ \\
\hline
\end{tabular}


Table III. Postoperative symptoms

\begin{tabular}{|lcc|}
\hline Symptom & Toupet & WTP \\
\hline TEM - transient esophageal motility & ++ & + \\
\hline Length of hospital stay & ++ & + \\
\hline Recovery room prolongation & + & - \\
\hline Dysphagia & - & - \\
\hline Vomiting & + - & - \\
\hline Coughing & ++ & + \\
\hline Bloating & ++ & + \\
\hline Swallowing disorders & - & - \\
\hline Sleeping disorders & + & $-/+$ \\
\hline IPP oral intake necessity & - & - \\
\hline
\end{tabular}

Table IV. Mean value $( \pm)$ of significant differences according to QoL questionnaire (scale 0-5 points, with $0=$ no symptoms and $5=$ advanced symptoms)

\begin{tabular}{|lcc|}
\hline Variable & WTP & Toupet \\
\hline Heartburn chronic & \pm 0.4 & \pm 1.0 \\
\hline Heartburn after meals & \pm 0.4 & \pm 1.0 \\
\hline Flatulence & \pm 1.2 & \pm 2.6 \\
\hline
\end{tabular}

5.4 days (2-16 post-operative days). As for patients after WTP the mean post-operative hospital stay was 4.7 days (2-9 post-operative days). No reoperations were necessary. No surgical complications were identified. As shown in Table III, patients following WTP had fewer minor symptoms of transient esophageal motility disorders after surgery, required shorter stay in the recovery room, more often denied dysphagia, experienced less coughing and vomiting, and had fewer swallowing difficulties and sleeping problems $(p<0.05)$. Thus the length of hospital stay was shorter.

We sent out the HRQoL questionnaires to all surgically treated patients. We received 96 (63.57\%) completed HRQoL questionnaires. The results were mainly positive in both groups. Statistically significant differences between the two groups of WTP and Toupet fundoplication patients were narrowed to answers to questions concerning firstly the presence of heartburn throughout the day. As presented in Table IV, the mean response in the WTP group was 0.4 points vs. 1.0 points in the Toupet group (where 0 points is asymptomatic, 1 point is a noticeable symptom but not disturbing). Secondly, another difference concerned the presence of heartburn after meals, where the response in the WTP group was 0.4 points vs. 1.0 point in the Toupet group (where 0 points is asymptomatic, 1 point is a noticeable symptom but not disturbing). Thirdly, a difference between the two groups was found for the question concerning the presence of flatulence. The WTP group gained on average 1.2 points vs. 2.6 points in the Toupet group (where 0 points means asymptomatic, 1 point is for a noticeable symptom but not disturbing, 2 points mean present but not every day, disturbing from time to time, and 3 points are for the symptom present every day). Differences between the two groups concerning other issues such as pain, discomfort following meals, discomfort during swallowing, regurgitation, amount of proton pump inhibitors (PPI) oral intake and general satisfaction after surgery were statistically insignificant factors.

\section{Discussion}

Fundoplication as a method of treatment should be performed in the safest possible way for the patient, and many factors should be taken into account such as peri-operative complications, hospital stay, long-term results and financial outcome [13]. We adopted the WTP procedure as it is a routine, well-practiced and safe method which overall gives fewer complications directly after surgery and at a later stage of convalescence. We consider the outcome of the WTP procedure as close to the natural topography of the stomach and esophagus as can be. With the realignment of the gastric fundus and the $90^{\circ}$ angle of His, which is as close to the natural physiology as possible, we achieve minimal post-operative inconvenience for patients. Bloating and flatulence and the ability to burp were practically eliminated. Signs of dysphagia during hospitalization and later on during outpatient clinic attendance were absent. Patients were discharged in good general condition with a high grade of satisfaction.

Whereas there is an abundance of data indicating post-operative complication rates ranging from $5 \%$ to $30 \%$ [13-16], our study shows that after the WTP procedure the rate was under $5 \%$. Throughout the 6-year observational period we did not observe in the WTP group any recurrences of hiatal hernia. Furthermore, no signs of esophageal temporary or persistent dysphagia were present; these are wellknown and identified symptoms of post-operative 
hiatal opening closures $[13,17,18]$. None of the patients in the WTP group were re-operated, but it is worth mentioning that in this group three patients formerly underwent a different cruroplasty and fundoplication (no. 1 = Dor, no. 2 = Nissen, no. 3 = Toupet operation). Currently these patients are in a satisfactory condition with no recurrent hiatal hernia or GERD. Although not always agreed on, according to our follow-up, the use of mesh reduces the rate of hiatal hernia recurrence [19-22]. Patients who underwent laparoscopic surgery were examined in the outpatient clinic, and we did not find any post-operative abdominal hernias in place of trocars in our material. Quality of life after minimally invasive surgery using the WTP procedure is highly favorable, with high satisfaction and practically immediate improvement after the operation. Ninety-one percent of WTP patients vs. $74 \%$ of the Toupet group stated in the HRQoL questionnaire that the operation helped them in such a way that, knowing what they know today and what awaits them after the procedure, they would, without a second thought, undergo the surgery once again.

Many authors have raised the possibility of erosion of the esophagus due to the use of polypropylene mesh placed under the esophagus on the crura and reinforced by suturing the esophageal hiatus. We did not observe any complications arising from this method, but we avoided direct contact of the sharp mesh edge with the esophagus in the hiatal opening. The mesh placed routinely in each patient has a protective value against tearing of the sutured crura of the diaphragm and hernia recurrence. This incorporation of the mesh into the crura is very easy due to the lack of serosal tissue covering the crural muscles during their dissection. The esophagus covered by a serous layer does not have the capability of adhesion to the mesh. We observed this phenomenon in 2 cases following a WTP; 1 patient had a gastrectomy and the other had a cholecystectomy at a later stage. This may imply that patients after WTP fundoplication can start physical effort much earlier then after traditional non-mesh techniques.

\section{Conclusions}

The minimally invasive WTP using mesh for the treatment of GERD and hiatal hernias gives longer awaited but satisfactory outcomes and optimistic long-term results. Due to the low percentage of post-operative complications, good quality of life of patients and a zero recurrence rate of hiatal hernia, this is a method of choice.

\section{Conflict of interest}

The authors declare no conflict of interest.

\section{References}

1. Wróblewski T, Grodzicki M, Ziarkiewicz-Wróblewska B, et al. Technical aspects of the posterior partial fundoplication - impact on the results of surgical treatment of gastroesophageal reflux. Videosurgery Miniinv 2006; 1: 6-9.

2. Kuster E, Ros E, Toledo-Pimentel V, et al. Predictive factors of the long term outcome in gastro-oesophageal reflux disease: six year follow up of 107 patients. Gut 1994; 35: 8-14.

3. Fuchs KH, Freys SM, Heimbucher J, et al. Pathophysiologic spectrum in patients with gastroesophageal reflux disease in a surgical Gl function laboratory. Dis Esophagus 1995; 8: 211-7.

4. Fein M, Ireland AP, Ritter MP, et al. Duodenogastric reflux potentiates the injurious effects of gastroesophageal reflux. J Gastrointest Surg 1997; 1: 27-33.

5. Lord RV, DeMeester SR, Peters JH, et al. Hiatal hernia, lower esophageal sphincter incompetence, and effectiveness of Nissen fundoplication in the spectrum of gastroesophageal reflux disease. J Gastrointest Surg 2009; 13: 602-10.

6. Wróblewski T, Grodzicki M, Ziarkiewicz-Wróblewska B, et al. Wyniki operacyjnego leczenia refluksu żołądkowo-przełykowego (GERD) sposobem laparoskopowym. Videochirurgia 2002; 7: 8-10.

7. Migaczewski M, Grzesiak-Kuik A, Pędziwiatr M, et al. Laparoscopic treatment of type III and IV hiatal hernia - authors' experience. Videosurgery Miniinv 2014; 9: 157-63.

8. Wróblewski T, Skalski M, Ziarkiewicz-Wróblewska B, et al. Progress in surgical treatment of reflux disease. Videosurgery Miniinv 2006; 1: 121-4.

9. Wróblewski T, Skalski M, Ziarkiewicz-Wróblewska B, et al. New antireflux surgical technique in GERD treatment. Videosurgery Miniinv 2007; 2: 139-44.

10. Piątkowski J, Jackowski M, Szeliga J. Laparoscopic surgery of esophageal hiatus hernia - single center experience. Videosurgery Miniinv 2014; 9: 13-7.

11. Fein M, Ritter M, DeMeester TR, et al. Role of lower esophageal sphincter and hiatal hernia in the pathogenesis of GERD. J Gastrointest Surg 1999; 3: 405-10.

12. Mattioli S, Lugaresi ML, Costantini M, et al. The short esophagus: intraoperative assessment of esophageal length. J Thorac Cardiovasc Surg 2008; 136: 834-41.

13. Stylopoulos N, Rattner DW, The history of hiatal hernia surgery: from Bowditch to laparoscopy. Ann Surg 2005; 241: 185-93.

14. Granderath FA, Schweiger UM, Pointner R. Laparoscopic antireflux surgery: tailoring the hiatal closure to the size of hiatal surface area. Surg Endosc 2007; 21: 542-8.

15. Rosenthal RJ, Jones DB. Laparoscopic hiatal hernia repair. Bariatric Times 2012; 9: 8-9. 
16. Jones MP, Sloan SS, Rabine JC. Hiatal hernia size is the dominant determinant of esophagitis presence and severity in gastroesophageal reflux disease. Am J Gastroenterol 2001; 96: 1711-7.

17. Hunter JG, Swanstrom L, Waring JP. Dysphagia after laparoscopic antireflux surgery. The impact of operative technique. Ann Surg 1997; 226: 109-10.

18. Hermann J, Kościński T, Malinger S, et al. Strangulation of the stomach and the transverse colon following laparoscopic esophageal hiatal hernia repair. Videosurgery Miniinv 2012; 7 : 311-4.

19. Kamolz T, Granderath FA, Bammer T, et al. Dysphagia and quality of life after laparoscopic Nissen fundoplication in patients with and without prosthetic reinforcement of the hiatal crura. Surg Endosc 2002; 16: 572-7.

20. Furnée E, Hazebroek E. Mesh in laparoscopic large hiatal hernia repair: a systematic review of the literature. Surg Endosc 2013; 27: 3998-4008.

21. Antoniou SA, Antoniou GA, Koch OO, et al. Lower recurrence rates after mesh-reinforced versus simple hiatal hernia repair: a meta-analysis of randomized trials. Surg Laparosc Endosc Percutan Tech 2012; 22: 498-502.

22. Schmidt E, Shaligram A, Reynoso JF, et al. Hiatal hernia repair with biologic mesh reinforcement reduces recurrence rate in small hiatal hernias. Dis Esophagus 2014; 27: 13-7.

Received: 11.03.2016, accepted: 25.03.2016. 